\title{
Single Chip Embedded System Solution: Efficient Resource Utilization by Interfacing LCD through Softcore Processor in Xilinx FPGA
}

\author{
Shiraz Afzal, Farrukh hafeez and Muhammad Ovais Akhter \\ Department of Electronic Engineering, Sir Syed University of Engineering and Technology, Karachi-Pakistan 75300 \\ Department of Electronic and Electrical Engineering Jubail Industrial College, Jubail- Saudiarabia \\ Department of Electronic Engineering, Sir Syed University of Engineering and Technology, Karachi-Pakistan 75300 \\ Email: safzal@ssuet.edu.pk,f_hafeez@jic.edu.sa, moakhter@ssuet.edu.pk
}

\begin{abstract}
This paper presents a customized application in term of LCD interfacing through soft-core microcontroller by implementing its control circuitry in FPGA. Therefore optimum efficiency is achieved. This provide the single chip solution by replacing conventional model which contain separate microcontroller circuit, therefore the efficient resource utilization of FPGA is made, day by day as an increase in the complexity of Embedded system design thus gives many advantages over conventional designed processors such as platform independence, less cost, flexibility and greater immunity to obsolescence.
\end{abstract}

Index Terms-FPGA, LCD interfacing, soft-core microcontroller e.t.c.

\section{INTRODUCTION}

A microcontroller is the major part of any embedded system that uses both hardware and software components to perform specific application. As embedded systems play a vital role in our everyday life also exist in profusion in our modern civilization and can be found in places such as in our vehicle, in health field, in industries and in amusement electronics to name just a few [1].

As an increase in complexity of embedded system. It became very difficult to design each hardware component because of the cost and become impractical approach therefore the emergence of Soft core microcontroller solve this problem in which a designer can easily build their design and verify that.

Here in this manuscript an important hardware element that relates to the embedded system which is LCD interfacing through soft-core controller is being controlled through Xilinx picoblaze controller as shown in Fig. 1.

The material of the manuscript is arranged as follow in section II explains the survey of softcore processors. Section III we describe the software used for HDL Language. Section IV describe the FPGA implementation which involves subsections as follow: Sub-section "a" describe the Pico-blaze Microcontroller Overview. Subsection "b" describes the VHDL component and design
Processes. Sub-section "c" describes the picoblaze instruction set and sub-section "d" describes the LCD display Overview.

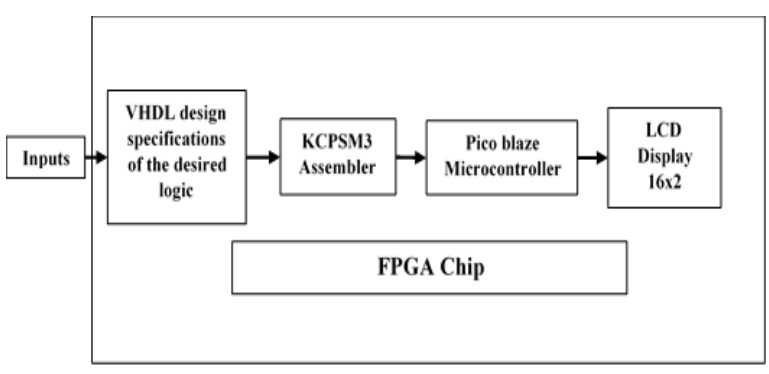

Fig.1. LCD interfacing through soft core processor

\section{REviEW OF AVAILABLE SOFT-CORE PROCESSORS}

This segment provide a list of available softcore processor specified by the both open source communities and commercial vendorsare provided

\section{A. Open Resource Softcores}

An open source community provides various cores freely available as given in [2]. These types of softcores are not only used for research purpose but also for the development of embedded systems [3], an example of such type of core used in academia for research purpose is UT Nios [4]. Another listof cores which is not only used forthe ASICs but can be also used for modeling and synthesize of FPGA is OpenSPARC [5]. Further LEON [6], LEON2 [6], LEON3 [5], Open RISC 1200 [2] and sun microsystem as provides theirown core are also available as open source soft cores. Details of which are available in [3].

B. Commercial Available Softcores:The major softcore controller includes Microblaze, Picoblaze by Xilinx, Nios II by Altera and Xtensa by Tensilica [3].

Microblaze and Picoblaze: This 32bit and 8bit softcore processor are incorporated by Xilinx for both Spartan and Virtex series, one of the leading vendor of FPGA. Microblaze is one of the demanding and mostly used 
softcore processor. It uses a Harvard RISC architecture, having 32 bit instruction set, and two level of interrupts and can operate up to $200 \mathrm{MHz}$ on Virtex 4 FPGAwhile Picoblaze is a compact 8 bit softcore controller can be useful for simple data processing application because of its miniature element and cost effective features [3].

Nios II by Altera: is a general purpose softcore processor that uses Harvard RISC architecture, 32 bit instruction set. It comes in three versions fast core, standard and economy further each core varies in performance and dimension depending on the chosen feature

\section{TOOL USED}

Xilinx $8.2 \mathrm{i}$ is used for the programming and configuring of FPGA, It give user friendly environment and can also provide flexibility in terms of by either implementing the logic using basic logical operator or with schematic diagram using schematic editor implementation.

Here in this task the LCD is programmed using VHDL language, further for picoblaze softcore processor KCPSM3 assembler is used. DOSBOX 0.74 is used for picoblaze psm file simulation.

\section{IMPLEMENTATION}

The work is carried out in the following segments:

A.Picoblaze Processor overview.

B. VHDL Components and design processes.

C.Picoblaze instruction set.

D. LCD display overview.

\section{A. Picoblaze Overview}

Fig. 2 shows the block diagram of picoblaze microcontroller, the significance of using picoblaze is of its small size, having RISC architecture and less FPGA slice available only 96 slices. It is given as a free, sourcelevel VHDL file with royalty-free re-use within Xilinx
FPGAs [8].

There main feature includes 64 bytes of internal memory, 256 input and output ports available, 16 byte wide general purpose register.

\section{B. VHDL Component and Design Processes}

VHDL components used by the picoblaze controller is shown in Fig. 3. They are mainly divide into two components that is block memory ROM and KCPSM3, KCPSM3 component provide the registers, Arithmetic logic unit etc.

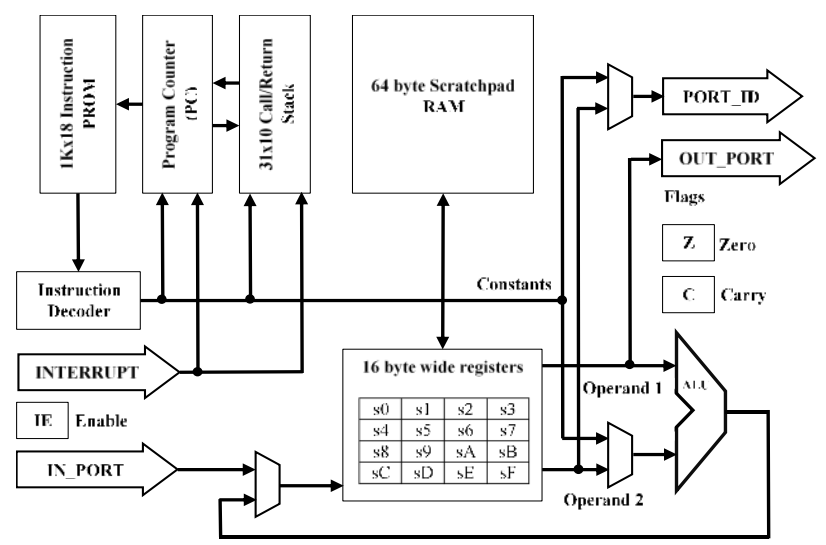

Fig.2. Block diagram of Picoblaze microcontroller

C. PicoblazeInstruction Set: The instructions mainly used by the picoblaze microcontroller is shown in table [3 manualref.].

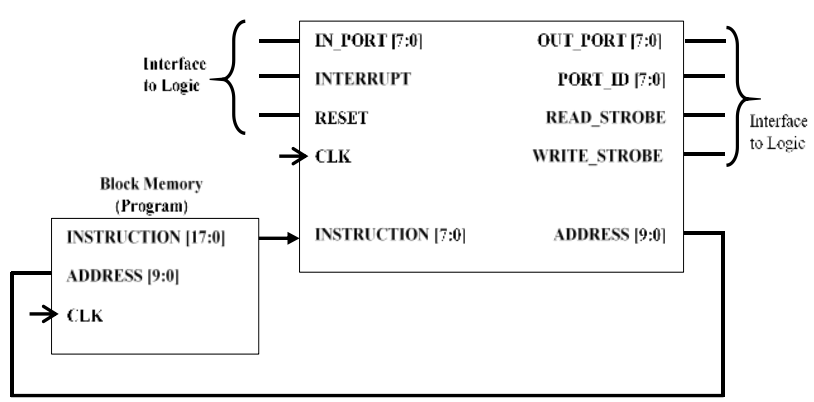

Fig.3. VHDL components used by the picoblaze controller 
Table 1. VHDL Instructions with Description

\begin{tabular}{|c|c|}
\hline Instruction & Description \\
\hline ADD aX, mm & Add register $\mathrm{aX}$ with literal $\mathrm{mm}$. \\
\hline ADD aX, bY & Add register aX with register bY. \\
\hline ADDCY aX, mm & Add register aX with literal $\mathrm{mm}$ with CARRY bit. \\
\hline ADDCY aX, bY & Add register aX with register bY with CARRY bit. \\
\hline AND aX, mm & Bitwise AND register aX with literal $\mathrm{mm}$. \\
\hline AND aX, bY & Bitwise AND register aX with register bY. \\
\hline CALL zzz & Unconditionally call subroutine at zzz. \\
\hline CALL C, zzz & If CARRY flag set, call subroutine at zzz. \\
\hline CALL NC, zzz & If CARRY flag not set, call subroutine at zzz. \\
\hline CALL NZ, zZZ & If ZERO flag not set, call subroutine at zzz. \\
\hline CALL Z, zZZ & If ZERO flag set, call subroutine at zzz. \\
\hline COMPARE aX, mm & $\begin{array}{l}\text { Compare register aX with literal mm Set CARRY and ZERO flags } \\
\text { asappropriate. Registers are unaffected. }\end{array}$ \\
\hline COMPARE aX, bY & $\begin{array}{l}\text { Compare register aX with register bY. Set CARRY and ZERO flags as } \\
\text { appropriate. Registers are unaffected }\end{array}$ \\
\hline DISABLE INTERRUPT & Disable interrupts. \\
\hline ENABLE INTERRUPT & Enable interrupts. \\
\hline FETCH aX, bY & Read scratchpad RAM location pointed to by register bY into register aX. \\
\hline FETCH aX, ss & Read scratchpad RAM location ss into register aX. \\
\hline INPUT aX, bY & Read value on input port pointed to by register bY into register aX. \\
\hline INPUT aX, pp & Read value on input port pp into register $\mathrm{aX}$. \\
\hline JUMP zzz & Unconditionally jump to zzz. \\
\hline JUMP C, zzz & If CARRY flag set, jump to aa. \\
\hline JUMP NC, zZZ & If CARRY flag not set, jump to aa. \\
\hline JUMP NZ, zZZ & If ZERO flag not set, jump to aa. \\
\hline JUMP Z, zzZ & If ZERO flag set, jump to aa. \\
\hline LOAD aX, mm & Load register aX with literal $\mathrm{mm}$. \\
\hline LOAD aX, bY & Load register aX with register bY. \\
\hline OR aX, mm & Bitwise OR register aX with literal $\mathrm{mm}$. \\
\hline XOR aX, bY & Bitwise XOR register aX with register bY. \\
\hline XOR aX, mm & Bitwise XOR register aX with literal $\mathrm{mm}$. \\
\hline TEST aX, bY & $\begin{array}{l}\text { Test bits in register aX against register bY. Update CARRY and ZEROflags. } \\
\text { Registers are unaffected. }\end{array}$ \\
\hline TEST aX, mm & $\begin{array}{l}\text { Test bits in register aX against literal } \mathrm{mm} \text {. Update CARRY and ZEROflags. } \\
\text { Registers are unaffected. }\end{array}$ \\
\hline SUBCY aX, bY & Subtract register bY from register aX with CARRY (borrow). \\
\hline SUBCY aX, mm & Subtract literal mm from literal aX with CARRY (borrow). \\
\hline SUB aX, bY & Subtract register bY from register aX. \\
\hline SUB aX, mm & Subtract literal $\mathrm{mm}$ from literal aX. \\
\hline STORE aX, ss & Write register aX to scratchpad RAM location ss. \\
\hline STORE aX, bY & Write register aX to scratchpad RAM location pointed to by register bY. \\
\hline SRX aX & Shift register aX right. Bit aX[7] is unaffected. \\
\hline SRA aX & Shift register aX right through all bits, including CARRY. \\
\hline SR1 aX & Shift register aX right, one fill. \\
\hline SR0 aX & Shift register aX right, zero fill. \\
\hline SLX aX & Shift register aX left. Bit aX[0] is unaffected. \\
\hline SLA aX & Shift register aX left through all bits, including CARRY. \\
\hline SL1 aX & Shift register aX left, one fill. \\
\hline SL0 aX & Shift register aX left, zero fill. \\
\hline $\mathrm{RR}$ aX & Rotate $\mathrm{aX}$ register right. \\
\hline RL aX & Rotate aX register left. \\
\hline RETURNI ENABLE & Return from interrupt service routine. Re-enable interrupt. \\
\hline RETURNI DISABLE & Return from interrupt service routine. Interrupt remains disabled. \\
\hline RETURN Z & If ZERO flag set, return from subroutine. \\
\hline RETURN NZ & If ZERO flag not set, return from subroutine. \\
\hline RETURN NC & If CARRY flag not set, return from subroutine \\
\hline RETURN C & If CARRY flag set, return from subroutine. \\
\hline RETURN & Return unconditionally from subroutine. \\
\hline OUTPUT aX, pp & Write register aX to output port location pp. \\
\hline
\end{tabular}




\section{LCD Display}

Spartan $3 \mathrm{E}$ uses $2 \times 16$ display consist of 2 lines of 16 character each. LCD is controlled by FPGA through an interface and also connected with Intel Startaflash whose function is to give full write/read access to LCD as shown in the Fig. 4.

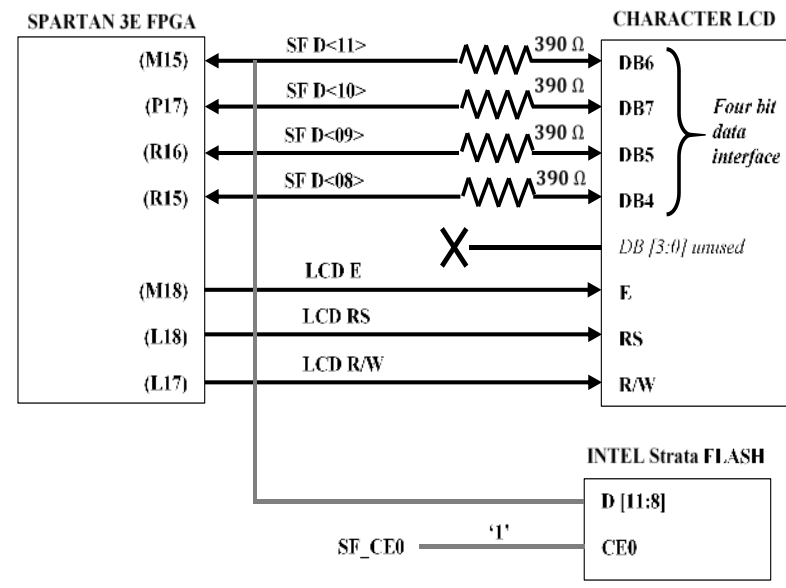

Fig.4. Read Write access to LCD

While the LCD display data ram locations shows in Fig. 5, only 32 characters can be displayed at a time.

Character Display Address

Undisplayed Address

\begin{tabular}{|c|c|c|c|c|c|c|c|c|c|c|c|c|c|c|c|c|}
\hline 00 & OI & 02 & 03 & 04 & $0 \mathbf{5}$ & & 07 & $t h$ & & $\mathbb{\|} \beta$ & $\mathrm{OB}$ & $\mathbb{N C}$ & & $\mathrm{OE}$ & & \\
\hline 40 & $\mathrm{Al}$ & 42 & 43 & H & 45 & 46 & 17 & 48 & 50 & 4. & $A B$ & AC & (I) & $4 \mathrm{E}$ & $\mathbb{A F}$ & \\
\hline
\end{tabular}

Fig.5. LCD Display

The commands to controlled LCD is sum up in the Table 2.

Table 2. Commands Controlled LCD

\begin{tabular}{|c|c|c|c|c|c|c|c|c|c|c|}
\hline \multirow[b]{2}{*}{ Function } & \multirow{2}{*}{ 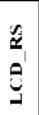 } & \multirow{2}{*}{ 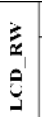 } & \multicolumn{4}{|c|}{ Upper Nibble } & \multicolumn{4}{|c|}{ Lower Nibble } \\
\hline & & & 总 & 参 & 㟧 & $\overrightarrow{\Delta \vec{\theta}}$ & $\hat{\tilde{\theta}}$ & 菊 & $\overline{\vec{\theta}}$ & 商 \\
\hline Clear Display & 0 & 0 & 0 & 0 & 0 & 0 & 0 & 0 & 0 & 1 \\
\hline Return Cursor Home & 0 & 0 & 0 & 0 & 0 & 0 & 0 & 0 & 1 & - \\
\hline Enter Mode set & 0 & 0 & 0 & 0 & 0 & 0 & 0 & 1 & 1D & $s$ \\
\hline Display 0n/Off & 0 & 0 & 0 & 0 & 0 & 0 & 1 & D & $\mathrm{C}$ & B \\
\hline cursor and Display Shift & 0 & 0 & 0 & 0 & 0 & 1 & $\mathrm{SC}$ & RL & - & - \\
\hline Function Set & 0 & 0 & 0 & 0 & 1 & 0 & 1 & 0 & - & - \\
\hline Set CGRAM Address & 0 & 0 & 0 & 1 & 15 & At & 43 & 12 & A1 & 10 \\
\hline Set DDRAM Address & 0 & 0 & 1 & 16 & 45 & A4 & 43 & 12 & A1 & 10 \\
\hline Read Busy Flag and Address & 0 & 1 & $\mathrm{BF}$ & 46 & 45 & A4 & 43 & 12 & A1 & 40 \\
\hline Write Data to CGRAM or DDRAYI & 1 & 0 & D? & D6 & D5 & D4 & D3 & $\mathrm{D} 2$ & D1 & $\mathrm{D} 0$ \\
\hline Read Data from CGRAM or DDRAM & 1 & 1 & D7 & D6 & D5 & D4 & D3 & D2 & D1 & D0 \\
\hline
\end{tabular}

The font bitmap for the characters stored in character generator ROM, It contains the Japanese kana characters and ASCII characters as shown in the Fig. 6.

In this assignment a KCPSM3 assembler is used which translates the input psm file and also three block RAM initialization templates and produces the output as shown in Fig. 7.

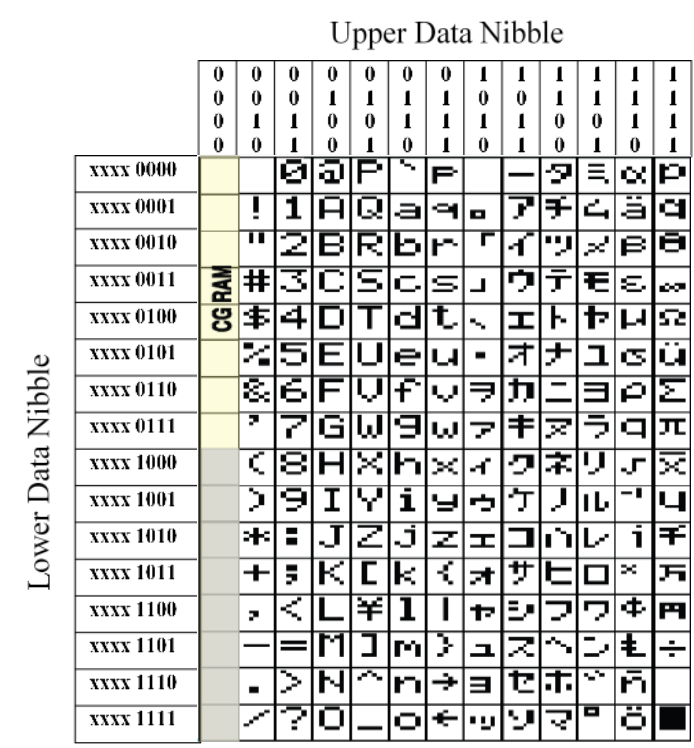

Fig.6. Font Bitmap Characters for LCD Display

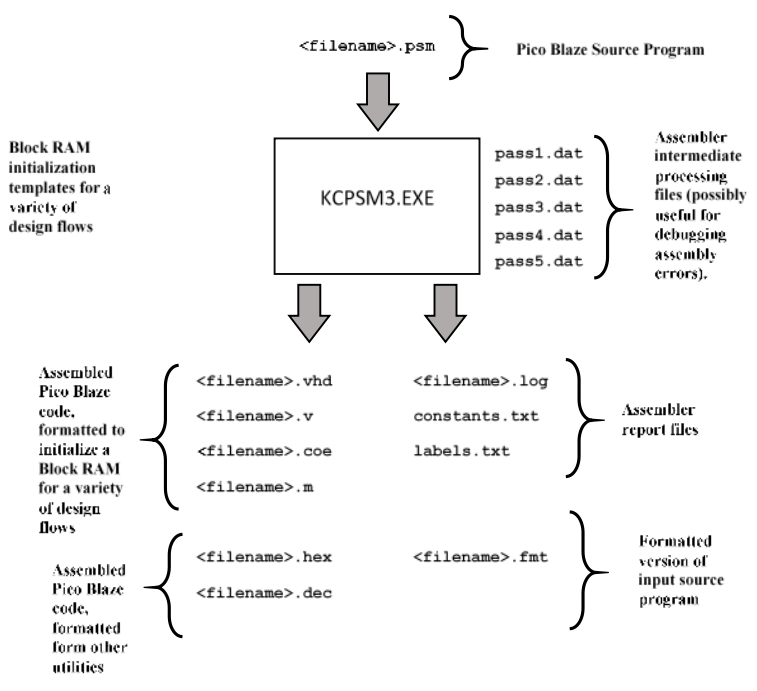

Fig.7. KCPSM3 Assembler

\section{Simulation RESUlts}

Fig. 9 shows the simulation result by displaying the name on the 2 line 16 character LCD using softcore picoblaze controller of Xilinx Spartan 3E FPGA.

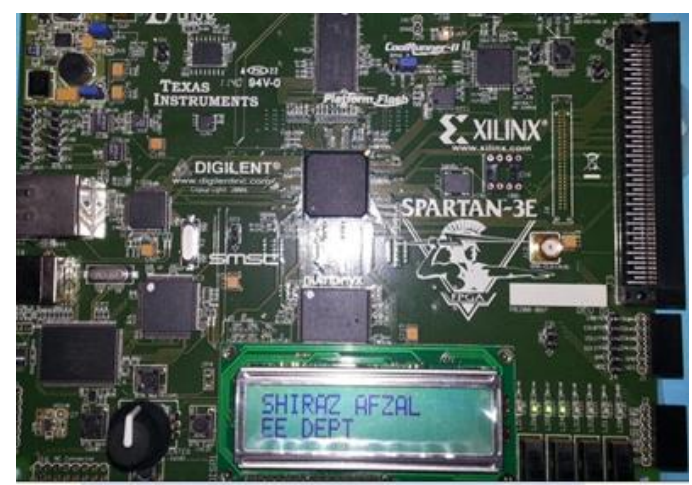

Fig.9. Result display on LCD 


\section{REFERENCES}

[1] Mishra, P. and Dutt, N., "Architectural description languages for programmableembedded system",IEE Proceedings of computer and digital technique, May 2005, pp. 285-297.

[2] Opencores.org Website, www.opencores.org, June 2006.

[3] Jason GT. Tong, Ian D. L Anderson and Muhammad A. S. Khalid, "Softcore processors for embedded system", IEEE Microelectronics International Conference, Dec 2006, pp. 170-173.

[4] UT Nios Homepage, www.eecg.toronto.edu/Uplavec/utnios.html, June, 2006.

[5] Open SPARC Website, www.opensparc.org, June 2006.

[6] "GRLIB IP Core User's Manual", Gaisler Research, February 2006.

[7] "LEON2 Processor User's Manual XST Edition", Gaisler Research, July 2005.

[8] PicoBlaze 8-bit Embedded Microcontroller User Guide, Online:

http://www.xilinx.com/support/documentation/ip_docume ntation/ug129.pdf, accessed 3 Dec 2012.

\section{Author's Profiles}

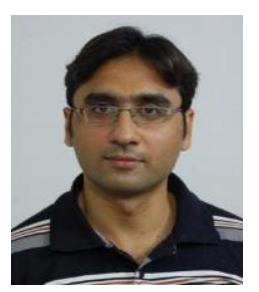

Shiraz Afzal is working as a Assistant Professor in Electronic Engineering Department at SSUET. He already published nine research articles in various International Journals. He has 8 years of teaching experience. He received his B.E. degree in Electronics from Sir Syed University of Engineering and Technology Karachi, Pakistan and M.E degree in Electronics, specialization in Micro-System design from NED University of Engineering \& Technology Karachi, Pakistan in 2006 and 2012 respectively. His research interest includes Microelectronic circuit design and Embedded System Design. He is a member of PEC.

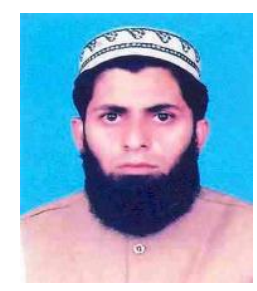

Farrukh Hafeez is working as a lecturer in Electrical and Electronic Engineering department at Jubail Industrial College. He received his B.E. degree in Electronics from NED University of Engineering and technology Karachi, Pakistan and M.E degree in Electronics, specialization in Industrial control system from NED University of Engineering \& Technology Karachi, Pakistan in 2005 and 2009 respectively. His research interest control system design and applications.

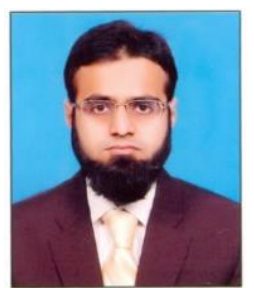

Muhammad Ovais Akhter is Assistant Professor in SSUET. Graduated in Electronic Engineering from same university. Over 6 years of experience in the area of Modeling, Simulation and Signal Processing.

How to cite this paper: Shiraz Afzal, Farrukh hafeez, Muhammad Ovais Akhter,"Single Chip Embedded System Solution: Efficient Resource Utilization by Interfacing LCD through Softcore Processor in Xilinx FPGA", IJIEEB, vol.7, no.6, pp.23-27, 2015. DOI: 10.5815/ijieeb.2015.06.04 